\title{
FLORÍSTICA DAS PRAIAS DA ILHA DE SÃO LUÍS, ESTADO DO MARANHÃO (BRASIL): DIVERSIDADE DE EŚPÉCIES E SUAS OCORRÊNCIAS NO LITORAL BRASILEIRO.
}

\author{
Maria Cristina C. CABRAL FREIRE, Reinaldo MONTEIRO ${ }^{i}$
}

\begin{abstract}
RESUMO - A Ilha de São Luís, situada ao norte do Estado do Maranhão, constitui-se numa região de transição entre duas floras distintas: flora amazônica e flora nordestina. Considerando esta peculiar situação fitogeográfica, o objetivo deste trabalho foi realizar o levantamento florístico nas prais arenosas da Ilha de São Luís e compaará-lo com os de outras áreas amostradas no litoral brasileiro. Foram totalizadas 260 espécies, compreendidas em 76 familias, sendo Fabaceae com o maior número de espécies (24). A comparação florística constatou que o estado da Bahia apresentou o maior número de espécies em comum com São Luís (63) e, em seguida, o estado do Pará (59). Dentre as espécies amostradas em São Luís, 125 foram exclusivas da região.
\end{abstract}

Palavras-chave: floristica, praia, diversidade.

Floristics of the Beaches of São Luís Island, State of Maranhão (Brazil): Species Diversity and Occurrence in the Brazilian Coast.

ABSTRACT - São Luis Island, located in the north of the State of Maranhão is itself a transitional region between the Amazonian and the northeastern floras. With such phytogeographical background, the aim of this paper was to realize a floristic survey on the sandy beaches of the São Luís Island and to compare this survey with other areas sampled along the Brazilian coast. A total of 260 species was found, distributed in 76 families, and among these the Fabaceae presenting the highest number of species (24). The floristic comparison showed that the State of Bahia has the highest number of species in common with São Luís (63), followed by the State of Pará (59). Among the species found in São Luis, 125 were exclusive of this area.

Key-words: floristics, beach, diversity.

\section{INTRODUÇÃO}

A vegetação litorânea ou justamarítima depende intimamente da natureza do substrato e da fisiografia. A diversidade morfológica mostrada nesta região, influenciada pela ação da água do mar e dos ventos constantes, propicia a formação de muitos habitats $\mathrm{e}$, consequentemente, o aparecimento de uma flora rica e variada (RIZZINI, 1979; ARAÚJO, 1984).
A palavra restinga tem sido utilizada para designar as formações vegetais litorâneas, englobando diversas comunidades como as da praia, antedunas, cordões arenosos, depressões entre cordões, margens de lagoas e até manguezais (LACERDA et al., 1982; ARAÚJO \& HENRIQUES, 1984). No sentido morfológico, as restingas têm sido consideradas como ilhas alongadas, faixas ou línguas de areias depositadas paralelamente ao

1 Departamento de Botânica, Instituto de Biociências, UNESP, Caixa Postal 199, 13506 Rio Claro, SP. 
litoral, graças ao dinamismo construtivo ou destrutivo das águas oceânicas (SUGUIU \& TESSLER, 1984).

RIZZINI (1979) mencionou três interpretações botânicas para as restingas brasileiras: formações vegetais que cobrem as areias holoceânicas desde o oceano, podendo alcançar as primeiras elevações da Serra do Mar; paisagem formada pelo areal justamarítimo com sua vegetação global e, finalmente, a mais comum, para indicar a vegetação lenhosa da parte interna, plana.

A heterogeneidade da região litorânea brasileira que se extende por aproximadamente $9000 \mathrm{~km}$, é evidenciada por todos os autores que se propuseram em subdividi-la. Uma das subdivisões mais recentes é apreșentada por SUGUIU \& TESSLER (1984), sendo baseada em elementos climáticos, oceanográficos e continentais. Nesta, o litoral está subdividido em: litoral amazônico ou equatorial (da foz do rio Oiapoque ao Maranhào oriental); litoral nordestino ou das barreiras (do Maranhão oriental ao recôncavo baiano); litoral oriental (do recôncavo baiano ao sul do Espírito Santo); litoral sudeste ou das escarpas cristalinas e litoral meridional ou subtropical (da região de Laguna à desembocadura do Arroio Chui). Toda esta diversidade ambiental (e climática) é refletida diretamente sobre a vegetação que se adapta às condições de cada área, havendo dificuldades naturais de se encontrar uma definição ampla e uma terminologia que se adequem a este ambiente costeiro.

Pela velocidade que vem se desenvolvendo a devastação na zona litorânea da Ilha de São Luís (MA) e, pela ausência de trabalhos no que diz respeito à vegetação das praias arenosas nesta região, é importante execução de trabalhos que diminuam a lacuna.

Por esta razào, FREIRE (1933) desenvolveu um trabalho sistemático de levantamento florístico na região litorânea da Ilha de São Luis, tendo abordado também aspectos de fenologia e distribuição dos hábitos das espécies nas diferentes regiões de coleta, segundo a topografía encontrada. Aqui são apresentados os resultados de composição florística das áreas de restinga amostradas, seguidos de comparação com outros levantamentos efetuados na costa brasileira.

\section{MATERIAL E MÉTODOS}

Caracterização da área de estudo

A Ilha de São Luís (2॰30'S; $44^{\circ} 16^{\prime} \mathrm{W}$ ) é politicamente dividida em três municipios: São José de Ribamar, Paço do Lumiar e, São Luís. É percorrida pelos rios Bacanga e Anil, que representam verdadeiros braços de mar devido à grande influência da água salgada recebida pelos mesmos. Além destes, pequenos cursos de água percorrem a Ilha, estando também sujeitos à ação periódica das marés. Ao longo dos rios encontram-se extensos manguezais.

A fisionomia do litoral da Ilha de São Luís pode ser descrita por duas faces distintas: a região norte que compreende um trecho de praias arenosas com dunas e pequenos cursos de água doce que 
apresentam manchas de mangue e, a região sul, caracterizada por inúmeros canais onde se desenvolvem exuberantes manguezais. Formações de falésias também estào presentes no trecho de praias arenosas.

A área escolhida para este estudo (FREIRE, 1993, Fig. 1) compreende o trecho entre as praias de Ponta D'Areia $\left(2^{\circ} 30,27^{\prime} S ; 4^{\circ} 19,08^{\prime} \mathrm{W}\right)$ e Araçagi $\left(2^{\circ} 27,55\right.$ 'S; $\left.44^{\circ} 10,13^{\prime} \mathrm{W}\right)$, correspondendo a aproximadamente $15 \mathrm{~km}$ de extensão, onde predominam as praias arenosas, com formações de dunas que podem alcançar alturas superiores a $30 \mathrm{~m}$. Esta área está inserida nos municípios de São Luís e Paço Lumiar (Praia de Araçagi) e está, em sua maior extensào próxima ao perimetro urbano e, por esta razão é bem frequentada e relativamente povoada.

\section{Coleta do material botânico}

Nos trechos delimitados para estudo, foram escolhidos oito áreas para amostragem da flora, que compreenderam retângulos de aproximadamente $50-150 \mathrm{~m}$ de largura, paralelos à linha de maré alta e com comprimento variando até $480 \mathrm{~m}$, dependendo da extensão das dunas.

As coletas foram mensais, durante 18 meses (de julho de 1988 a dezembro de 1989), abrangendo somente a flora fanerorgânica. O material coletado foi identificado com auxilio de bibliografia especializada e de taxonomistas de instituições nacionais, e está depositado nos herbários do Departamento de Biologia da Universidade Federal do Maranhào e do Instituto de Biociências da UNESP, campus de Rio Claro (HRCB).

O sistema de classificação utilizado foi o de CRONQUIST (1988).

As espécies encontradas foram listadas e comparadas com listagens fornecidas pela literatura para os Estados do Pará (SANTOS \& ROSÁRIO, 1988; BASTOS, 1988 e LISBOS et al., 1993), Alagoas (SILVA, 1972 e ESTEVES, 1978), Pernambuco (LIMA, 1951), Rio Grande do Norte (TAVARES, 1960 e TRINDADE, 1982), Bahia (SEABRA, 1949 e PINTO et al., 1984), Rio de Janeiro (HAY et al., 1984 e ARAÚJO \& HENRIQUES, 1984), São Paulo (ANDRADE \& LAMBERTI, 1965 e DE GRANDE \& LOPES, 1981), Rio Grande do Sul (RAMBO, 1954; PFANDENHAUER, 1978 e CORDAZZO \& SEELIGER, 1989) e Santa Catarina (BRESSOLIN, 1979 e CORDAZZO \& COSTA, 1989).

Outras fontes bibliográficas foram consultadas para melhor abrangência das distribuições geográficas das espécies encontradas na Ilha de São Lùís (FLORA ILUSTRADA DE SANTA CATARINA - REITZ, 19651989, e diversos números da FLORA NEOTROPICA). No entanto, para efeitos de comparação e elaboração da Figura 3, somente foram consideradas as obras acima relacionadas pois citam especificamente a ocorrência das espécies no mesmo ambiente de coleta deste trabalho.

\section{RESULTADOS E DISCUSSÃO}

\section{Material botânico}

Foram totalizadas 260 espécies 


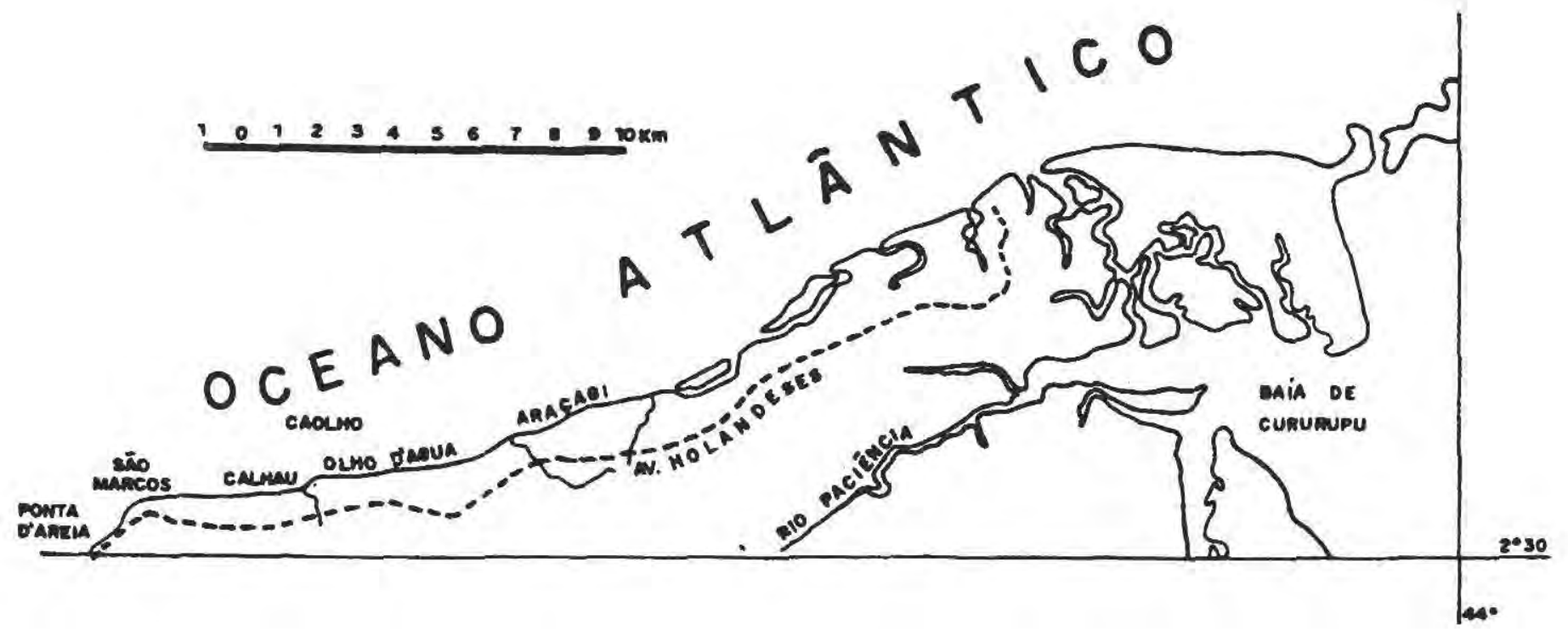

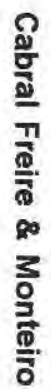

Figura 1. Localização das áreas de amostragem (praias) na Ilha de São Luís (MA), Brasil. 
nas áreas de amostragem, compreendidas em 78 familias (Tab. 1). As Dicotiledôneas (Magnoliopsida) foram as mais representativas, com 220 espécies $(84,62 \%)$, distribuídas em 62 famílias. As Monocotiledôneas (Liliopsida) apresentaram 40 espécies $(15,38 \%)$, distribuídas em 14 familias.

A familia Fabaceae apresentou o maior número de espécies (24) dentre as Dicotiledôneas (Fig. 2), seguida de Mimosaceae (12), Caesalpiniaceae (11) e Myrtaceae (10). Entre as Monocotiledôneas (Fig. 2), Poaceae foi a mais numerosa, com 12 espécies, seguida por Cyperaceae (8).

Além das Poaceae e Cyperaceae, espécies consideradas neste levantamento são frequentes em ambientes ruderais da Ilha de São Luís. Estas espécies são encontradas em locais onde a luminosidade é acentuada, como nas dunas, além de pequenas clareiras em áreas de vegetação densa, próximas às praias (FREIRE, 1993).

O total de espécies demonstra a flora diversificada desta região que se distribui ao longo das dunas, em zonas de fisionomias bastante distintas. A riqueza desta flora reflete, sem dúvida, a localização intermediária da Ilha de São Luis, entre os litorais amazônico e nordestino, que apresentam clima, geologia e flora tão distintos. Desta forma, as espécies que ocorrem na Ilha, além daquelas encontradas exclusivamente ali, incluem muitas comuns ao litoral amazônico e/ou nordestino.

A proximidade com a área urbana e a frequência de banhistas nas áreas de amostragem, devem ter proporcionado a entrada de espécies atípicas de praias, como as ruderais. Mais, certamente, este fato não comprometeu

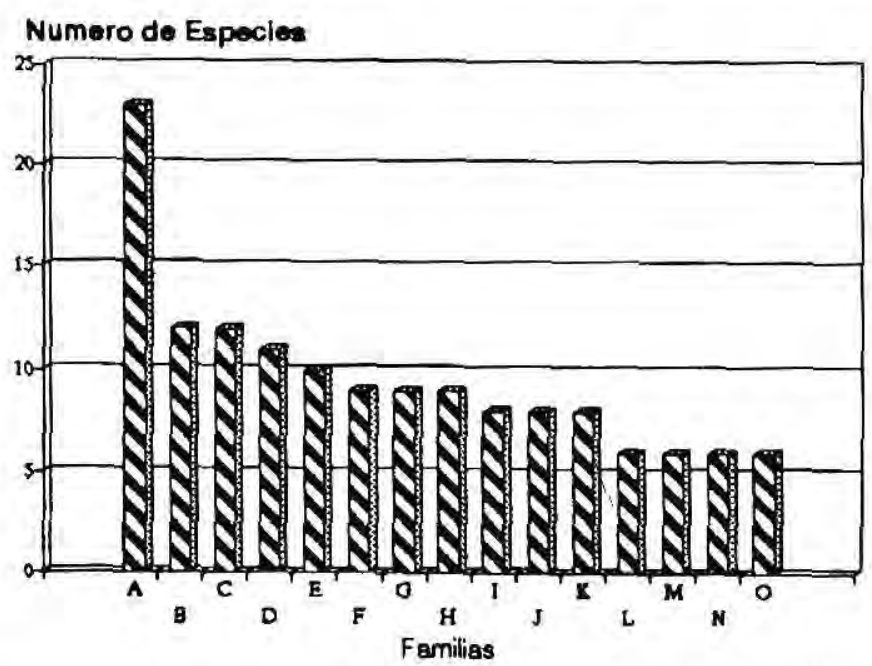

Figura 2. Familias mais frequentes nas áreas de amostragem no litoral da Ilha de São Luis (MA), Brasil. A - Fabaceae; B - Mimosaceae; C - Poaceae; D - Caesalpiniaceae; E Myrtaceae; F - Asteraceae; G - Euphorbiaceae; H - Solanaceae; I - Cyperaceae; J Malpighiaceae; K - Rubiaceae; L - Apocynaceae; M - Convolvulaceae; N - Malvaceae; $\mathrm{O}$ - Melastomataceae. 
Tabela 1. Lista das espécies ocorrentes no litoral da Ilha de São Luís. estado do Maranhão (Brasi) e suas ocorrência no litoral brasileiro. Abreviaturas para os estados na coluna ocorrência: $\mathrm{AC}=$ Acre, $\mathrm{AL}=$ Alagoas, $\mathrm{AM}=$ Amazonas, $\mathrm{AP}=$ Amapá, $\mathrm{BA}=$ Bahia, $\mathrm{CE}-$ Ceará, $\mathrm{DF}$ $=$ Distrito federal, $\mathrm{ES}=$ Espirito Santo, $\mathrm{GO}=$ Goiás, $\mathrm{MG}$ - Minas Gerais, $\mathrm{MT}=$ Mato Grosso, $\mathrm{Pa}=$ Pará, $\mathrm{PB}=$ Paraiba, $\mathrm{PE}=$ Pernambuco, $\mathrm{PI}=$ Piauí, $\mathrm{RJ}=$ Rio de Janeiro, $\mathrm{RN}=$ Rio Grande do Norte, $\mathrm{RO}=$ Rondônia, $\mathrm{RS}=$ Rio Grande do Sul, $\mathrm{SC}=$ Santa Catarina, $\mathrm{SP}=$ São Paulo.

\begin{tabular}{ll}
\hline Familia/Espécie & Ocorrência \\
\hline
\end{tabular}

\section{Magnoliopsida}

Aizoaceae

Sesuvium portulancastrum L. HRCB 12673)

Amaranthaceae

Alternanthera brasiliana (L.) O. Kuntze (HRCB 10670)

Altemanthera maritima (Mart.). St. Hil (HRCB 9943)

Bitutaparon portulacoides (St. Hil.) Mears (HRCB 13444)

Anacadiaceae

Anacardium occidentale L. (HRCB 12671)

Spondias futea L. (HRCB 12672)

Annonaceae

Rollinia exsucca (DC ex. Dunal). A. DC. (HRCB 10663)

Apocynaceae

Hymatanthus articulata (Vahl.). Woodson (HRCB 10458)

Hymatanthus sp. (HRCB 10457

Mandevilla hirsuta (A. Rich.). K. Sch. (HRCB 10552)

Mandevilla scabra (R. \& S.). K. Sch. (HRCB 10550)

Mandevilla sp. (HRCB 10553)

Rhabdadenia biflora (Jacq.). Muell. Arg. (HRCB 10551)

Asclepiadaceae

Asciepias curassavica L. (HRCB 9961)

Calotropis procera (Ait.) R. Br. (HRCB 12670)

Matelea maritima (Jacq.) Woodson (HRCB 11571)

Oxypetalum sp. (HRCB 11570)

Oxypetalum banksii Roem. \& Schultes (HRCB 10664)

Aristolochiaceae

Aristolochia sp. (HRCB 13203)

Asteraceae

Ambrosia microcephala DC. (HRCB 9949)

Eclipta alba (L.) Hassk. (HRCB 9947)

Eleutheranthera ruderalis Schultz (HRCB 9950)

Emilia sonchifolia L. (HRCB 12684)

Eupatorium sp. (HRCB 9948)

Mikania cordifolia Wiild. (HRCB 9944)

Vemonia helophilla Mart. (HRCB 9960)

Vemonia scorpioides (Lam.) Pers. (HRCB 11255)

Wedelia hookeriana Gardin (HRCB 13199)

Bignoniaceae

Anemopaegma parkeri Spague (HRCB 11573)

Arrabidaea coleocalyx Burt et K. Sch. (HRCB 9959)

Cydista aequinoctialis (L.) Miers (HRCB 11572)

$P A, P E, A L, B A, R J, S C, R S$

RJ

$P E, A L, B A, R J$

$R N, P E, A L, B A, R J, S P, P R, S C, R S$

$P A, R N, A L, B A, R J, A M, C E, D F, E S, G O$, MT, MG, PB, PE, PI, SC, SP

PA, AM

PA, AM

PA, SP, AM

$P A, B A, S P, A M$

$B A, S P, R S$

$P E, A L, B A$

RJ

RJ

$A L, R S$

PA, AL, BA

RJ, RS

RJ, SP, RS

Memora navida (DC.) Bur e K. Schum. S. L. (HRCB 11570)

Phryganocidia corymbosa Bur. (HRCB 12679)

Bomnacaceae

Pseudobombax grandiflora (Cav.) A. Robins (HRCB 11622)

Boraginaceae

Cordia multispicata Cham. (HRCB 12000)

PA

BA, RJ

RJ, SP

Cordia sp. (HRCB 11612) 
Cont.

\begin{tabular}{|c|c|}
\hline Família/Espécie & Ocorrência \\
\hline Hellotropium polyphyllum Lehem. (HRCB 13463) & PA \\
\hline Toumefortia bicolor Sw. (HRCB 12676) & SP, AM, PA, BA, SC, RJ \\
\hline \multicolumn{2}{|l|}{ Toumefortla volubills $\mathrm{L}$. )HRCB 12005) } \\
\hline \multicolumn{2}{|l|}{ Chrysobalanaceae } \\
\hline Chrysobalanus Icaco L. (HRCB 11611) & $P A, R N, A L, B A, R J, S P, S C$ \\
\hline $\begin{array}{l}\text { Hyrtella racemosa Lam. var, hexandra (Willd. ex. R. \& S.) Prance } \\
\text { (HRCB 10661) }\end{array}$ & PA \\
\hline \multicolumn{2}{|l|}{ Clusiaceae } \\
\hline Vismia guianensis Aubl. (HRCB 9927) & PA \\
\hline \multicolumn{2}{|l|}{ Connaraceae } \\
\hline Connarus favosus Planch. (HRCB 11998) & AM, CE, MT, PA \\
\hline \multicolumn{2}{|l|}{ Connanus sp. (HRCB 11625) } \\
\hline \multicolumn{2}{|l|}{ Rourea sp. (HRCB 11623) } \\
\hline Convolvulaceae & \\
\hline Ipomoea bahiensis Willd. ex R. \& Sch. (HRCB 9928) & BA \\
\hline
\end{tabular}

Ipomoea nil (L.) Roth. (HRCB 9931)

ipomoea pes-caprae (L.) Sweet. (HRCB 9948)

Ipomoea stolonifera (Cyrill.) Gmelin (HRCB 9945)

Ipomoea sp. (HRCB 9959)

$R N, P E, A L, B A, R J, S P, S C$

$P A, R N, A L, B A, R J, S P, S C$

Merremia aegyptia (L.) Urban (HRCB 9953)

Cucurbitaceae

Momordica charantia L. (HRCB 11578)

AL, BA

Dichapetalaceae

Dichapetalum vestifum Baill. (HRCB 11997)

Dilleniaceae

Curatelkla americana L. (HRCB 11631)

Davilla nexuosa St. Hil. (HRCB 11649)

BA, PA

Euphorbiaceae

Chamaesyce hyssopifolia (L.) Small (HRCB 11575)

Cnidosculus urens (L.) Arthur (HRCB 11577)

$P A, A L, B A$

Croton glandulosus $L$. var. hirtus (L Herit) Muell. Arg. (HRCB 11576) PA, AM, BA, RJ, SP, PR, SC

Dalechampia scandens L. (HRCB 9956)

Hyeronima alchameoides Fr. Allem. (HRCB 11643)

Manihot guinguepartita Huber ex Rogers \& App. (HRCB 10660)

Margaritaria nobilis L. F. (HRCB 11640)

Ricinus comunis L. (HRCB 9908)

Sapium glandulatum (Vell.) Pax (HRCB 11577)

Fabaceae

Abrus precatorius L. (HRCB 12687)

Calerogonium mucunoides Deauv. (HRCB 9908)

Canavalia obtusifolla DC. (HRCB 9955)

Centrosema arenarium Benth. (HRCB 12090)

Centrosema brasiliensis (L.) Benth. (HRCB 10503)

Centrosema pubescens Benth. (HRCB 12012)

AM, PA, PI, CE, RN, BA, ES, RJ, SP, PR, SC $P A$

$P A, S C, P R, S P, R J, E S, B A$

$P E, A L, S P, S C$

RJ

$R N, A L, B A, R J, A M, R O$

BA

RN, PE, RJ, SP, SC

$P A, R N, P E, A L, B A$

PA

Clitoria guianensis Benth, var. guianensis (HRCB 10525)

Ciitoria falcata Lam, var. faicata (HRCB 10526)

Crotalaria retusa L. (HRCB 10504)

Dalbergia ecastophyllum (L.) Taubert (HRCB 10527)

Dalbergia sp. (HRCB 11646)

Desmodium barbatum (L.) Benth. (HRCB 11250)

Dloclea grandifiora Mart. (HRCB 11251)

Dioclea virgata (Rich.) Amsh. (HRCB 11237)

indigofera micocarpa Desv. (HRCB 12694)

$P E, A L, B A$

$P A, R N, A L, B A, R J, S P, R S$

PA

AL 
Cont.

Familia/Espécie

Ocorrência

Machaerium aculeatum Raddi (HRCB 11239)

RJ

Macroptilium Jathyroides L. (HRCB 12689)

Mucuna urens DC. (Sloan.) Fawc. \& Rendler (HRCB 10522)

Ormosia sp. (HRCB 12691)

Phaseolus sp. (HRCB 11249)

Rhyncosia phaseo/oides (Sw.) DC. (HRCB 11242)

Stylosanthes guianensis Sw. (HRCB 13442)

Zomia dyphylla Pers. (HRCB 13445)

Flacourtiaceae

Banara guianensis Aubl. (HRCB 12688)

Casearia grandiflora Camb. (HRCB 11244)

Gentianaceae

Schultesia stenophylfa Mart. (HRCB 13447)

Lamiaceae

Lamium sp. (HRCB 10028)

Masyplanthes chamaedrys (Vahl) Kuntz (HRCB 9931)

Lauraceae

Cassytha americana Ness (HRCB 13450)

Ocotea sp. (HRCB 11579)

Lecythidaceae

Gustavia augusra L. (HRCB 11616)

Lecythis lurida (Miers) Mori (HRCB 12016)

Lecythis sp. (HRCB 11619)

Loranthaceae

Psitacanthus dichrous Mart. (HRCB 11628)

Loganiaceae

Spigelia anthelmia L. (HRCB 10561)

Malpighiaceae

Banisteriopsis pubipetala (Juss.) Cuatr. (HRCB 11581)

Malpigiaceae

Bunchosia fluminensis Griseb. (HRCB 11247)

Byrsonima crassifolia (L.) Kunth (HRCB 12681)

Byrsonima sp. (HRCB 12008)

Heteropteris anoptera Juss. (HRCB 12680)

Tetrapterys ovalifolia Grisebach (HRCB 12004)

Tetrapterys maranhamensis Juss. (HRCB 13202)

Tetrapterys sp. (HRCB 12001)

Malvaceae

Hibiscus dimidiatus Schrank (HRCB 11998)

Hibiscus tiliaceus L. (HRCB 10530)

Pavonia cancellata Cav. (HRCB 11583)

Pavonia malacophylla (Link et Otto) Garke (HRCB 12685)

Sida cordifolia L. (HRCB 10531)

Urena lobata L. (HRCB 9957)

Marcgraviaceae

Noranthea brasiliensis Choisy (HRCB 10514)

Melastomataceae

Clidemia hirta (L.) Don (HRCB 12691)

BA

$P A, B A, R J$

$P A, R N, A L, B A, S P, R S, A M$

AM, AP, AC, RO

$P A, A M, A P, P E, M T, G O, M G, S P$

PA, RJ, ES, SC

BA, RJ

PA, PE, BA, RJ, AM, SC, SP

$P A, A M, A P, R O$

$B A, P A, A M, R O, A C$

$B A, R J$

AL

PA, RO, AM, PI, CE, MT, GO, MG, RJ, SP, PR

PA

\author{
$A L, S P, R S$ \\ $R N, A L, B A$ \\ RJ \\ $B A, R J$ \\ $R N, A L, B A, S P$ \\ BA, RJ, SP \\ BA \\ $B A$ \\ AM, RO, PA, RR, AP \\ $A M, A C, P A, G O, A P$ \\ $A M, P A, A C, A P, R O$
}

Pterolepis sp. (HRCB 11584) 
Cont.

\begin{tabular}{|c|c|}
\hline Família/Espécie & Ocorrência \\
\hline \multicolumn{2}{|l|}{ Menispermaceae } \\
\hline Odontocarya duckei Barneby (HRCB 11634) & PA \\
\hline \multicolumn{2}{|l|}{ Mimosaceae } \\
\hline Entada polyphylla Benth. (HRCB 10656) & PA, AM \\
\hline Inga bahiensis Benth. (HRCB 11596) & PA \\
\hline \multicolumn{2}{|l|}{ Inga setifera DC. (HRCB 11253) } \\
\hline Inga thibaudiana DC. (HRCB 11252) & PA \\
\hline \multicolumn{2}{|l|}{ Inga sp. 1 (HRCB 13453) } \\
\hline \multicolumn{2}{|l|}{ Inga sp. 2 (HRCB 10657) } \\
\hline \multicolumn{2}{|l|}{ Mimosa caesalpiniifolia Benth. (HRCB 10665) } \\
\hline Mimosa invisa Mart. (HRCB 10666) & RS \\
\hline \multicolumn{2}{|l|}{ Mimosa velloziana Mart. (HRCB 11243) } \\
\hline Pithecellobium cochleatum Mart. (HRCB 10670) & PA \\
\hline \multicolumn{2}{|l|}{ Pithecellobium sp, 1 (HRCB 10671) } \\
\hline \multicolumn{2}{|l|}{ Pithecellobium sp. 2 (HRCB 10664) } \\
\hline \multicolumn{2}{|l|}{ Moraceae } \\
\hline Ficus catappifolia Kunth. \& Bouché (HRCB 11245) & $P A, A L, R J$ \\
\hline Ficus guianensis Dev. in Ham. (HRCB 11244) & $\mathrm{PA}, \mathrm{AM}, \mathrm{RO}, \mathrm{AP}, \mathrm{GO}$ \\
\hline \multicolumn{2}{|l|}{ Myrtaceae } \\
\hline Calycolpus goetheanus (DC.) Berg (HRCB 10517) & PA \\
\hline Eugenia bihora (L.) DC. (HRCB 9945) & PA \\
\hline Eugenia patrisii Vahl (HRCB 9947) & PA \\
\hline Eugenia punicifolia (HBK) DC. (HRCB 9948) & PA \\
\hline \multicolumn{2}{|l|}{ Eugenia tapacumensis Berg (HRCB 10332) } \\
\hline \multicolumn{2}{|l|}{ Eugenia sp. 1 (HRCB 9953) } \\
\hline \multicolumn{2}{|l|}{ Eugenia sp. 2 (HRCB 9957) } \\
\hline Myrcia cuprea (Berg) Kiaresk. (HRCB 10516) & PA \\
\hline Myrcia multifora (Lam.) DC. (HRCB 9960) & RJ, SC \\
\hline \multicolumn{2}{|l|}{ Psidium sp. (HRCB 13457) } \\
\hline \multicolumn{2}{|l|}{ Nyctaginaceae } \\
\hline \multicolumn{2}{|c|}{ Guapira pernambucensis (Casar.) Lindeli (HRCB 10519) } \\
\hline \multicolumn{2}{|l|}{ Ochnaceae } \\
\hline Ouratea fieldigiana (Gard.) Engl. (HRCB 13455) & BA \\
\hline \multicolumn{2}{|l|}{ Ouratea sp. (HRCB 13454) } \\
\hline Sauvagesia erecta L. (HRCB 11632) & $P A, R J, S P, R S, A M$ \\
\hline \multicolumn{2}{|l|}{ Olacaceae } \\
\hline Dulacia candida (Poeppig) Kuntz (HRCB 11630) & AC, AM, ES, MT, PA, RO \\
\hline \multicolumn{2}{|l|}{ Onagraceae } \\
\hline Luowigia affins (Jacq.) Rav. (HRCB 9955) & \\
\hline Ludwigia hyssofolia (G. Don.) Exell. (HRCB 9959) & PA, AM \\
\hline Passifloraceae & \\
\hline Passinora coccinea Aubl. (HRCB 13456) & AM \\
\hline Passinora foetida L. (HRCB 13450) & BA \\
\hline Passiffora nitida HBK. (HRCB 13451) & $P A, B A$ \\
\hline Plumbaginaceae & \\
\hline Plumbago scandens L. (HRCB 12686) & AL, RJ \\
\hline Polygalaceae & \\
\hline Bredemeyera sp. (HRCB 11647) & \\
\hline Polygala monticula HBK. (HRCB 13458) & \\
\hline Securidaca bialata Benth. (HRCB 11595) & \\
\hline Polygonaceae & \\
\hline Caccoloba latifolia Lam. (HRCB 11627) & PA, BA, RJ \\
\hline Rubiaceae & \\
\hline Bomerla verticilata (L.) GFW. Mey (HRCB 13460) & PA, PE, AL, SP \\
\hline
\end{tabular}


Cont.

\begin{tabular}{|c|c|}
\hline Familia/Espécie & Ocorrência \\
\hline Chiococca alba (L.) Hitch. (HRCB 10658) & AL, BA, RJ, SP \\
\hline \multicolumn{2}{|l|}{ Chomelia obtusa Cham. \& Schlecht (HRCB 11615) } \\
\hline Guettarda angelica Mart. ex Muell Arg. (HRCB 12677) & BA \\
\hline \multirow{2}{*}{\multicolumn{2}{|c|}{$\begin{array}{l}\text { Guettarda platypoda DC. (HRCB 11613) } \\
\text { Isertia speciformis DC. (HRCB 10515) }\end{array}$}} \\
\hline & \\
\hline Tocoyena brasiliensis Mart. (HRCB 11246) & BA \\
\hline \multicolumn{2}{|l|}{ Rutaceae } \\
\hline & AM, PA, RN, BA, RJ, SC, SP, PR \\
\hline Matayba guianensis Aubl. (HRCB 11642) & PA, BA, RJ, SP \\
\hline \multicolumn{2}{|l|}{ Matayba sp. (HRCB 11620) } \\
\hline Pseudima frutescens (Aubl.) Radlk. (HRCB 9961) & PA \\
\hline \multicolumn{2}{|l|}{$\begin{array}{l}\text { Sapotaceae } \\
\text { Franchetella catocladantha Eichler (HRCB 9957) }\end{array}$} \\
\hline \multicolumn{2}{|l|}{ Franchetella catocladantha Eichler (HRCB 9957) } \\
\hline Manilkara subsericea (Mart.) Dubard (HRCB 11633) & RJ, PR, SC, SP \\
\hline \multicolumn{2}{|l|}{ Manilkara sp. (HRCB 11614) } \\
\hline \multirow{2}{*}{\multicolumn{2}{|c|}{$\begin{array}{l}\text { Sideroxylan sp. (HRCB 11644) } \\
\text { Scrophulariaceae }\end{array}$}} \\
\hline & \\
\hline Scoparia dulcis L. (HRCB 11648) & $A L, B A, S P, R S$ \\
\hline \multicolumn{2}{|l|}{ Solanaceae } \\
\hline \multicolumn{2}{|l|}{ Cestrum sp. (HRCB 10559) } \\
\hline Solanum aculeatissimum Jacq. (HRCB 13453) & AL \\
\hline Solanum americanum Mill. (HRCB 13452) & RN, SP, RS, SC, PR, ES, RJ \\
\hline \multicolumn{2}{|l|}{ Solanum grandifforum Ruiz et Pav. (HRCB 10542) } \\
\hline Solanum juripeba L. C. Rich. (HRCB 10545) & $\begin{array}{l}\text { PA } \\
\text { RN PE, AL, RJ ES SP PR SC }\end{array}$ \\
\hline Solanum panicufatum L. (HRCB 10553) & RN, PE, AL, RJ, ES, SP, PR, SC \\
\hline \multicolumn{2}{|l|}{ Solanum sp. 1 (HRCB 10546) } \\
\hline Physalls angulata L. (HRCB 10660) & BA \\
\hline \multicolumn{2}{|l|}{ Sterculiaceae } \\
\hline Guazuma u/mifolia Lam. (HRCB 10662) & PA \\
\hline \multicolumn{2}{|l|}{ Guazuma sp. (HRCB 10874) } \\
\hline \multicolumn{2}{|l|}{ Sterculiaceae } \\
\hline \multicolumn{2}{|l|}{ Helicteris sp. (HRCB 10876) } \\
\hline \multirow{2}{*}{\multicolumn{2}{|c|}{$\begin{array}{l}\text { Waltheria viscosissima St. Hil. (HRCB 12003) } \\
\text { Tiliaceae }\end{array}$}} \\
\hline & \\
\hline Apeiba tibourbou Aubl. (HRCB 9958) & BA \\
\hline \multicolumn{2}{|l|}{ Turneraceae } \\
\hline \multicolumn{2}{|l|}{ Piriqueta viscosa Griseb. (HRCB 11248) } \\
\hline Tumera uimifolia L. (HRCB 9951) & $R N, P E, A L, R J$ \\
\hline Tumera sp. (HRCB 10870) & \\
\hline Ulmaceae & \\
\hline Trema micrantha Miq. (HRCB 13448) & BA, SP, RS \\
\hline Verbenaceae & \\
\hline Amasonia sp. (HRCB 11246) & \\
\hline Lippia sp. (HRCB 11248) & \\
\hline Lantana camara L. (HRCB 11250) & $A L, R J, S P, R S$ \\
\hline Lantana sp. (HRCB 11255) & \\
\hline Violaceae & \\
\hline Hybanthus ipecacuanha Baill. (HRCB 10662) & RN, BA \\
\hline Vitaceae & \\
\hline Cissus erosa Rich. (HRCB 11239) & PA \\
\hline Cissus sicyoldes L. (HRCB 11241) & PA, RJ, SP, RS \\
\hline Cissus sp. (HRCB 11626) & \\
\hline
\end{tabular}


Cont.

Familia/Espécie

Ocorrência

Liliopsida

Araceae

Anthurium sinuatum Benth. ex Schott (HRCB 11561)

PA

Dracotium asperum C. Koch (HRCB 11563)

Philodendron scandens C. Koch \& H. Sello (HRCB 11562)

Philodendron sp. (HRCB 11563)

Arecaceae

Astrocaryum tucuma Mart. (HRCB 11596)

Diphothemium campestre Mart. (HRCB 11594)

Euterpe oleracea Mart. (HRCB 11590)

Mauritia nexuosa L. f. (HRCB 11581)

Orbygnia phalerata Mart. (HRCB 11595)

Bromeliaceae

Bromelia sp. (HRCB 11582)

Commelinaceae

Commelina virginica $L$. (HRCB 11574)

Dichorisandra tenuir Mart. (HRCB 10669)

Cyperaceae

Cyperus densicaespitosus Mattf \& Kunkenth (HRCB 10545)

Cyperus ligularis L. (HRCB 10546)

Cyperus luzulae (L.) Retz. (HRCB 10547)

Cyperus surinamensis Rottb. (HRCB 10549)

Cyperus sp. 1 (HRCB 13201)

Cyperus sp. 2 (HRCB 13205)

Eleocharis geniculata (L.) R. \& S. (HRCB 13206)

Fimbristylis spathacea Roth. (HRCB 10522)

Eriocaulaceae

Paepalanthus polytricoides Kunth (HRCB 12009)

Heliconiaceae

Heliconia psittacorum L. f. (HRCB 10521)

Maranthaceae

Calathea lindbergii Peters (HRCB 11564)

Orchidaceae

Oeceoclades maculata (Lindl.) Lindl. (HRCB 11565)

Poaceae

Andropogon teucostachyus HBK. (HRCB 10543)

Cenchrus echinatus L. (HRCB 13438)

Dactyloctenium aegyptium (L.) Beauv. (HRCB 13463)

Eragrostis ciliaris (L.) R. Br. (HRCB 10553)

Panicum trichoides Swartz (HRCB 11629)

Paspalum maritimum Trin. (HRCB 10541)

Paspalum millegrana Scharder (HRCB 11567)

Paspalum arenarium Scharder (HRCB 16०30)

Scleria sp. (HRCB 11630)

Setaria vulpiseta (Lam.) Roem. et Schult. (HRCB 11566)

Sporobulus virginicus (L.) Kunth (HRCB 13440)

Streptostachys asperifolia Desv. (HRCB 13201)

Smilacaceae

Smilax schomburgkiana Kunth. (HRCB 11636)

Xyridaceae

Xyris anceps Lam. (HRCB 13449)

Zingiberaceae

Costus arabicus L. (HRCB 10523)

PA

RS

$P A, R N, A L$

BA, SC, RS

$P A, R N, A L, R J$

BA

Monocotiledônea Indeterminada

MC n 151) 
o número de espécies encontradas, pois estas representaram apenas $10 \%$ do total (Tab. 1).

As vegetações herbácea e rasteira da região próxima à linha de maré e a lenhosa das regiões subsequentes, são bastante distintas do tipo descrito por BRAGA (1979), para a restinga da Amazônia. Na Ilha de São Luís, estas duas vegetações cobriram as areias em verdadeiros emaranhados, principalmente na época das chuvas. A vegetação lenhosa apresentou árvores que alcançaram mais de $15 \mathrm{~m}$ de altura e, em muitos locais apresentavam aspecto de uma floresta.

Para BRAGA (1979), a restinga da Amazônia apresenta uma biomassa mediocre, de fisionomia uniforme e com penetração de luz excessiva. SANTOS \& ROSÁRIO (1988) discordam desta observação, com base em levantamentos feitos na vegetação ficadora de dunas no Pará.
Espécies tidas como típicas da vegetação de dunas e restingas das regiões Norte e Nordeste estão presentes na Ilha de São Luiș, como Anacardium occidentale, Blutaparon portulacoides, Byrsonima crassifolia, Canavalia obtusifolia, Cereus fernambucensis, Chrysobalanum icaco, Dalbergia ecastophyllum, Eugenia biflora, Hibiscus tiliaceus, Hybanthus ipecacuanha, Hymatanthus articulatus, Ipomoea pescaprae, Myrcia cuprea, Sporobolus virginicus, etc (SAMPAIO, 1945; PIRES, 1973; BRASIL, 1973; ESTEVES, 1978; BRAGA, 1979). Este fato evidencia a influência das flores destas regiões na Ilha de São Luis (Fig. 3).

Para RAWISTCHER (1944), Hibiscus tiliaceus é um "companheiro acidental dos litorais limosos", o que foi comprovado para presença desta espécie apenas nas áreas com manchas de mangue.

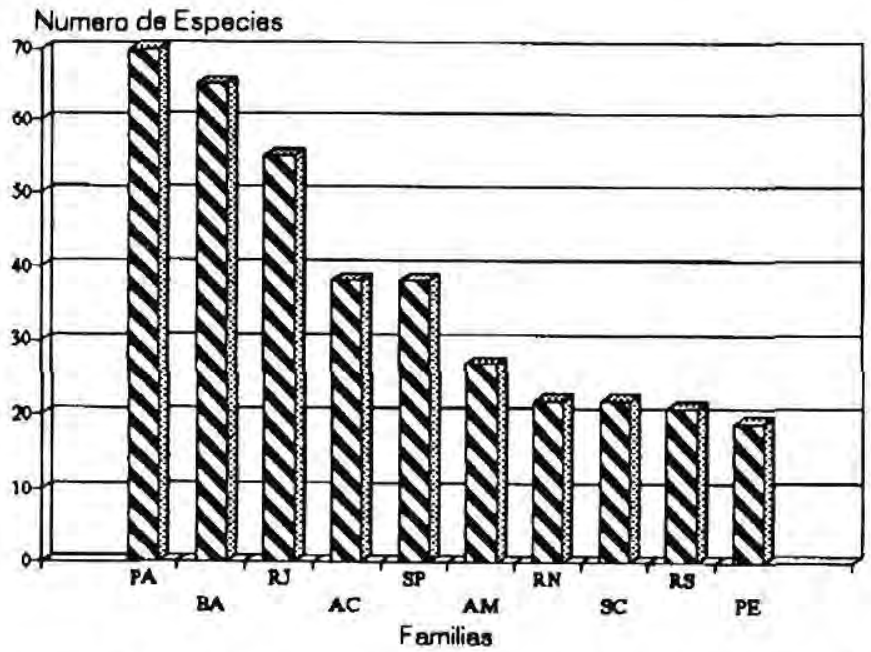

Figura 3. Número de espécies de vegetação litorânea comuns entre a Ilha de São Luís (MA) e outros estados brasileiros. 
o número de espécies encontradas, pois estas representaram apenas $10 \%$ do total (Tab. 1).

As vegetações herbácea e rasteira da região próxima à linha de maré e a lenhosa das regiões subsequentes, são bastante distintas do tipo descrito por BRAGA (1979), para a restinga da Amazônia. Na Ilha de São Luís, estas duas vegetações cobriram as areias em verdadeiros emaranhados, principalmente na época das chuvas. A vegetação lenhosa apresentou árvores que alcançaram mais de $15 \mathrm{~m}$ de altura e, em muitos locais apresentavam aspecto de uma floresta.

Para BRAGA (1979), a restinga da Amazônia apresenta uma biomassa medíocre, de fisionomia uniforme e com penetração de luz excessiva. SANTOS \& ROSÁRIO (1988) discordam desta observação, com base em levantamentos feitos na vegetação ficadora de dunas no Pará.
Espécies tidas como típicas da vegetação de dunas e restingas das regiões Norte e Nordeste estão presentes na Ilha de São Luís, como Anacardium occidentale, Blutaparon portulacoides, Byrsonima crassifolia, Canavalia obtusifolia, Cereus fernambucensis, Chrysobalanum icaco, Dalbergia ecastophyllum, Eugenia biflora, Hibiscus tiliaceus, Hybanthus ipecacuanha, Hymatanthus articulatus, Ipomoea pescaprae, Myrcia cuprea, Sporobolus virginicus, etc (SAMPAIO, 1945; PIRES, 1973; BRASIL, 1973; ESTEVES, 1978; BRAGA, 1979). Este fato evidencia a influência das flores destas regiões na Ilha de São Luis (Fig, 3).

Para RAWISTCHER (1944), Hibiscus tiliaceus é um "companheiro acidental dos litorais limosos", o que foi comprovado para presença desta espécie apenas nas áreas com manchas de mangue.

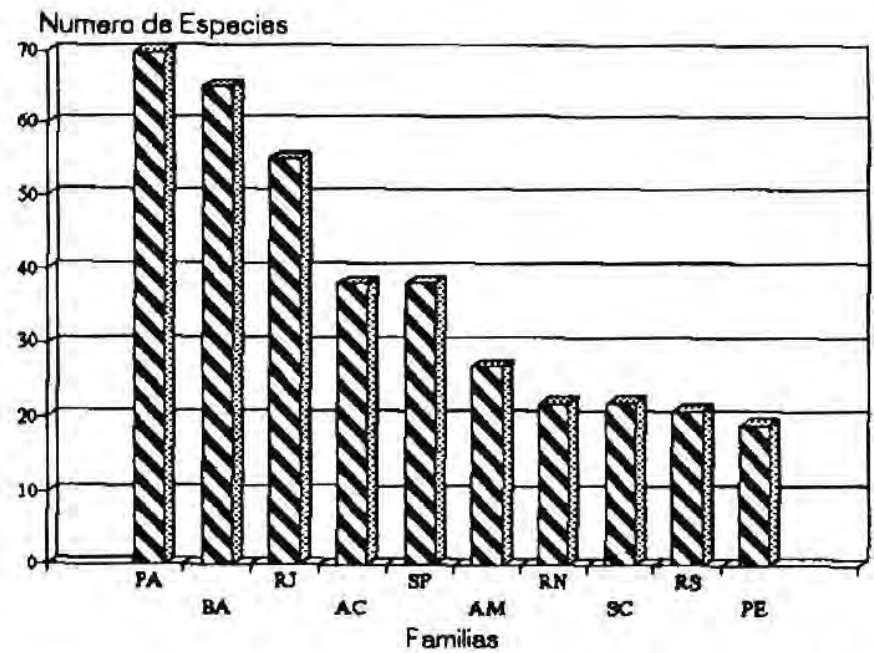

Figura 3. Número de espécies de vegetação litorânea comuns entre a Ilha de São Luís (MA) e outros estados brasileiros. 
Entre as espécies coletadas na Ilha de São Luís, encontram-se madeiras de importância econômica para a região, como Gustavia augusta, Hymenaea sp. e Ocotea sp. (CARDOSO, 1983), bem como frutas comestiveis típicas da Amazônia, tais como Anacardium occidentale (caju), Byrsonima crassifolia (murici), Euterpe oleracea (açai), Mauritia flexuosa (buriti) e Spondias lutea (cajá) (CAVALCANTE, 1974).

Espécies características do cerrado como Curatella americana e Orbignya phalerata, e da caatinga como Mimosa caesalpiniifolia (BRASIL, 1973), foram também amostradas na Illha de São Luís.

Entre as 62 famílias encontradas, as Leguminosas (Caesalpiniaceae, Fabaceae e Mimosaceae) foram as mais frequentes, totalizando 47 espécies. Em seguida, a familia Poaceae, com 12 espécies e Myrtaceae, com 10 espécies. Estas famílias apresentam também uma boa representatividade nos diversos trechos do litoral brasileiro, como mostram os diversos estudos realizados nestes ecossistemas.

RAMBO (1954) e LINDEMAN et al. (1975) citaram para o litoral riograndense as Asteraceae, Cyperaceae e Solanaceae. Estas familias tiveram também uma boa representatividade nos levantamentos de REITZ (1961) para Santa Catarina e DE GRANDE \& LOPES (1981) para a Ilha do Cardoso (SP).

No Estado do Rio de Janeiro, ARAÚJO \& HENRIQUES (1984) destacaram as Leguminosas, Rubiaceae,
Orchidaceae, Myrtaceae, Poaceae e Bromeliaceae. ARAÚJO \& OLIVEIRA (1988) destacaram, particularmente para a Ilha Grande (RJ), as Leguminosas, Rubiaceae, Orchidaceae e Bromeliaceae.

Para as regiões Norte e Nordeste, a maioria das famílias encontradas no litoral do Rio de Janeiro, Rio Grande do Sul, Santa Catarina e São Paulo, também foram representativas, como mostraram os levantamentos feitos por ESTEVES (1978) em Alagoas, PINTO et al. (1984) no litoral baiano e, SANTOS \& ROSÁRIO (1988) no Pará.

De fato, a maioria das familias citadas anteriormente são também frequentes na Ilha de São Luís. Muitas destas, como as Leguminosas, Poaceae e Rubiaceae, são também representativas em outros ecossistemas. devido inclusive ao grande número de especies que apresentam em regiões tropicais. Convém ressaltar, entretanto, que as familias Bromeliaceae e Orchidaceae são muito pouco frequentes na Ilha de São Luís, apresentando uma espécie cada uma destas. Pelo levantamento realizado, por SANTOS \& ROSÁRIO (1988), este fato também foi observado no Pará.

Similaridade florística com outros Estados

A região Nordeste brasileira, representada nos levantamentos florísticos do litoral pelos Estados de Alagoas, Bahia, Pernambuco e Rio Grande do Norte, apresentou maior número de espécies em comum com a Ilha de São Luis, que também faz parte desta região geográfica (Fig. 3 e Tab. 1). Além das semelhanças fisionômicas, deve-se levar em consideração que estes estados 
apresentam clima semelhante, ou seja, pequena variação de temperatura anual e períodos de chuva e seca bem definidos durante $o$ ano. Além disso, a proximidade destas áreas, que apresentam substrato semelhante, favorecem a colonização por espécies já adaptadas a estas condições ambientais.

Um outro fator que deve ser levado em consideração ao analisarmos as semelhanças flor iticas entre os Estados é a intensidade de coletas realizadas nestas áreas, principalmente no Estado da Bahia. Este Estado apresentou um maior número de áreas amostradas o que, sem dúvida, refletiu no número de espécies coletadas.

Comparando as listas florísticas do estado da Bahia com a apresentada por este trabalho, encontram-se 63 espécies em comum (Tab. 1). Embora estas áreas apresentem semelhanças fisionômicas e clímáticas, como já ditas anteriormente, a distância entre São Luís e a Bahia é grande quando comparada com os demais estados nordestinos, que não apresentaram tantas espécies comuns pela própria escassez de estudos.

O Estado do Pará, geograficamente o mais próximo da Ilha de São Luís, apresentou 59 espécies em comum (Fig. 3, Tab. 1), número inferior ao apresentado em relação ao Estado da Bahia. Entretanto, ocorreram 30 espécies exclusivas ao Estado do Pará e a Ilha de São Luís. Similarmente ao litoral maranhense, o litoral do Estado do Pará sofre influência da flora amazônica e desembocadura dos grandes rios da regiâo. Este fato volta a chamar atenção quanto à intensidade de coletas, pois assim como no Estado do Maranhão, no Pará foram poucos os estudos realizados na flora litorânea, sendo estes os únicos em termos de região Norte, já que para o Amapá não houve qualquer referência.

O Rio de Janeiro, terceiro Estado em número de espécies comuns à Ilha de São Luis, é exemplo de área de intensas coletas na região litorânea. Foram encontradas 54 espécies em comum (Fig. 3, Tab. 1), sendo nove exclusivas. Além das diferenças climáticas e estruturais entre estas duas áreas, a distância entre estas deve ser levada em consideração na análise da similaridade florística. A maioria das espécies comuns a estas duas áreas não são típicas apenas de dunas, pois ocorrem na maior parte do litoral brasileiro, em outros ambientes.

As semelhanças florísticas entre o litoral fluminense e as áreas de São Luís existem, mas poderiam tomar uma menor proporção caso fossem realizadas coletas mais intensas nas áreas próximas à Itha de São Luís, principalmente nos Estados do Pará, Piauí e Ceará.

As semelhanças fisionômicas observadas entre São Luis e algumas áreas do Estado de Santa catarina não refletiram no número de espécies comuns a estes locais (Fig. 3, Tab. 1). As diferenças climáticas e a localização geográfica dos mesmos, certamente foram fatores que influenciaram nas diferenças florísticas encontradas. As poucas espécies em comum entre São Luis e Santa Catarina, sem dúvida, ocorrem em função do substrato e 
relevo semelhantes, que acabam por favorecer a colonização de espécies frequentes em ambientes de dunas, com adaptações às pressões ambientais encontradas em cada área.

Pela própria condição de região de transição em que se encontra São Luís, já se poderia prever uma flora diversificada, com a influência das floras adjacentes. Entretanto, só haverá uma análise mais consistente sobre a similaridade florística entre as áreas do litoral, quando se tiver uma boa parte destes ecossistemas estudados.

\section{Bibliografia Citada}

ANDRADE, M. A. B. \& LAMBERTI, A. 1965. A vegetação. In: AZEVEDO, A, $A$ haixada santista: aspectos geográficos. 1a. ed. Sảo Paulo. Ed. Universidade de São Paulo, v.1, p.151-200.

ARAÚJO, D. S. D. 1984. Comunidades vegetais. In: LACERDA, L. D. ; ARAÚJJO, D.S.D; MACIEL, N.C. (orgs.). Restingas: origem, estrutura e processos. Niterói, CEUFF, $216 \mathrm{p}$.

ARAÚJO, D. S. D. \& HENRIQUES, R. P. B. 1984. Análise florística das restingas do estado do Rio de Janeiro. In: LACERDA, L. D. ; ARAÚJO, D.S.D; MACIEL, N.C.(orgs.). Restingas: origem, estrutura e processos. Niterói. CEUF. p.159-193.

ARAÚJO, D. S. D. \& OLIVEIRA, R R. DE. 1988. Reserva Biológica Estadual da Praia do Sul (Ilha Grande, estado do Rio de Janeiro): lista preliminar da flora. Acta Botanica Brasileira, 1(supl.)(2):8394.

BASTOS, M. DE N. DO C. 1988. Levantamento floristico em restinga arenosa litorânea da Illha de Maiandeua - Pará. Boletim do Museu Paraense Emilio Goeldi, 4(1):159-172.
BRAGA, P. I. S. 1979. Subdivisào fitogeográfica, tipos de vegetação, conservação e inventário florístico da floresta amazônica. Acta Amazonica, 9(supl.)(4):53-80.

BRASIL. 1973. Departamento Nacional de Produção Mineral. Projeto RADAM, Folha SA23 São Luis e parte da Folha SA24 Fortaleza. Rio de Janeiro. s.p.

BRESSOLIN, A. 1979. Flora da restinga da Ilha de Santa Catarina. Insula, 10:1-54.

CARDOSO, N. 1983, Madeiras da Amazônia. Manaus. Instituto de Tecnologia da Amazônia - UTAM. 12p.

CAVALCANTE, P, B. 1974. Frutas comestiveis da Amazônia. II. Publicações avulsas do Museu Paraense Emilio Goeldi, 27:9-12.

CORDAZZO, C. V \& COSTA, C. S. B. 1989. Assoiações vegetais das dunas frontais de Garopaba (SC). Ciencia e Cultura, 41(9):906-910.

CORDAZZO, V. C. \& SEELIGER. V. 1987. Composição e distribuição nas dunas costeiras ao sul de Rio Grande (RS). Ciéncia e Cultura, 39(3):321-324

CRONQUIST, A. 1988. The evolution and classification of flowering plants. 2 ed. New York. New York Botanical Garden. $555 \mathrm{p}$.

DE GRANIDE, D. A. \& LOPES, E. 1981. Plantas da restinga da Ilha do Cardoso (São Paulo, Brasil), Hochnea, 9:1-22.

ESTEVES, G. L. 1978. Contribuição ao conhecimento da vegetação da restinga de Macéio, Macéio, Secretaria de Planejamento do Estado de Alagoas Coordenação do Meio Ambiente. 42p.

FREIRE, M. C. C. M. 1993. Estudos floristicos na região litorânea da Ilha de São Luis (MA). Dissertação Mestrado, Instituto de Biociência, UNESP, Rio Claro, SP. $142 p$.

HAY, J. D. V.; HENRIQUES, R. P. B.; LIMA, D. M. 1984. Quantitative comparisons of dune and foredune vegetation in restinga ecosystems in the State of Rio de Janeiro, Brazil. Revista Brasileira de Biologia, 41(3):655-662. 
LACERDA, L. D.; ARAÚJO, D. S. D.; MACIEL, N. C. 1982. Restingas brasileiras: uma bibliografia. Rio de Janeiro, Fundação José Bonifácio. 56p.

LIMA, D. DE A. 1951. A flora de Boa Viagem. Boletim da SAIC, 18(1/2):121-125.

LINDEMAN, J. C. et al. 1975. Estudos botânicos no Parque Estadual de Torres, Rio Grande do Sul, Brasil: levantamento florístico da Planície do Cortume, da área de Itapeva e da área Colonizada. Iheringia (Ser. Bot.),21:15-52.

LISBOA, P. L. B.; LISBOA, R. C. L; ROSA, N. DE A.; SANTOS, M. R. DOS. 1993. Padrões de diversidade florística na Reserva Ecológica do Bacurizal, em Salvaterra. Ilha do Marajó, Pará. Boletim do Museu Paraense Emilio Goeldi, série botânica, 9(1)[no prelo].

PFANDENHAUER, J. 1978. Contribuição ao conhecimento da vegetação e de suas condições de crescimento nas dunas costeiras do Rio Grande do Sul, Brasil. Revista Brasileira de Biologia, 38(4):827-836.

PINTO, G. L. P.; BAUTISTA, H. P; FERREIRA, J. D. C. A. 1984. A restinga do litoral nordeste do Estado da Bahia. In: LACERDA, L.D; ARAÚJO, D.S.D.; MACIEL, N.C. (orgs.). Restingas: origem, estrutura e processos. Niterói, CEUFF. p.195-216.

PIRES, J. M. 1973. Tipos de vegetação da Amazônia. Publicações Avulsas do Museu Paraense Emilio Goeldi, Belém, 20:179-202.

RAMBO, R. 1954. História da flora do litoral riograndense. Sellowia, 6:113-172.

RAWITSCHER, F. K. 1944. Algumas noções sobre a vegetação do litoral brasileiro. Boletim da Associação de Geógrafos Brasileiros, 5:13-28.
REITW, P. R. 1961. Vegetação da zona marítima de Santa Catarina. Sellowia, 13:17-155.

REITW, P. R. (ed.). 1965-1989. Flora Ilustrada Catarinense. Itajaí, Herbário Barbosa Rodrigies. 149 monografias.

RIZZINI, C. T. 1979. Tratado de fitogeografia do Brasil: aspectos sociológicos e florísticos. 2a. ed. Sào Paulo, HUCITEC. 374p.

SAMPAIO, A. J. 1945. Observações botânicas. III. Avenidas naturais na vegetação do litotal. Chácaras e Quintais, 1(6):418-419.

SANTOS, J. V. M. DOS \& ROSÁRIO, C. DA S. 1988. Levantamento da vegetação fixadora das dunas de Algodoal-PA. Boletim do Museu Paraense Emilio Goeldi (Sér. Bot.), 4(1):133-154.

SEABRA, J. J. DE A. 1949. Flora das dunas (apontamentos sobre a flora psamófila das dunas de Itapoã-Bahia). Lilloa, 20:187-192.

SILVA, M. DE A. Flora das praias de Macéio. Anais do ICB-UFRPE, 2(2):111-121.

SUGUIU, K. \& TESSLER, M. G. 1984. Planicies de cordões litorâneos quaternários do Brasil: origem e nomenclatura. In: LACERDA, L. D.; ARAÚJO, D, S. D.; MACIEL, N. C. (orgs.), Restingas: origem, estrutura e processos. Niterói, CEUFF. p.15-25.

TAVARES, S. 1960. Estudos geobotânicos no Rio Grande do Norte. Arquivos do Institulo de Pesquisas Agronômicas, 5:39-51.

TRINDADE, A. 1982. Plantas fixadoras de dunas via costeira-Natal, RN. Coleção Textos Acadêmicos (UFRN). p, 1-37. 